the behaviour of the structure modelled is predicted. On the basis of these predictions, the final structure is designed."

The introductory lecture by R. E. Rowe was entitled: "Model Analysis and Testing--its Relation to Established Methods of Design and its Accuracy in Predicting the Behaviour of the Actual Structure". B. C. Best described "Certain Aspects of Model Testing for the Cumberland Basin Scheme". G. Somerville discussed "The Model Test of the Manchester Skyway Bridge". "The Model Test of the Smithfield Market Shell Roof" was the subject of the lecture by L. L. Jones. G. D. Base contributed a paper on "The Model Test of the Structure of the Metropolitan Cathedral of Christ the King". "The Cost of Model Testing and the Time Required" was the subject of a second lecture by R. E. Rowe.

A summary of the conclusions arising from this meeting, at which considerable interest was shown in the papers presented, may be stated as follows: (I) Model analysis and testing have now reached a stage where they can be considered as a design technique in its own right which can be used to predict the actual behaviour of reinforced and pre-stressed concrete structures reliably and accurately. (2) Cost and time required for model testing are comparable with those associated with established design techniques. (3) Future developments in the use of model testing are likely to be considerable in providing a means of defining the appropriate structural form as well as providing the relevant design information for that form. It is envisaged that a number of models will be used for any given project. (4) The availability of this technique should free the designer from the need to utilize structural forms lying within the scope of analytical procedures, enabling him to design rationally and economically the imaginative structures such as eity redevelopments as foreseen in the Buchanan Report.

An appendix describes briefly the work of the Istituto Sperimentale Modelli e Strutture (I.S.M.E.S.), Bergamo, Italy, officially set up in 1951 to provide a model testing service primarily for dams and other hydroelectric structures in that country.

\title{
FORMATION OF RAINDROPS
}

$\mathrm{T}$ $\mathrm{HE}$ condensation process, the three-phase (or Bergeron-Findeisen) process, and the collection process, it is generally believed, are the three processes which lead to the growth of water-drops in a cloud and eventually to the formation of rain. Only small droplets are initiated by the condensation process, but the other two processes are sufficiently rapid to result in the formation of raindrops.

U. Shafrir and M. Neiburger, in the first of the series of Occasional Papers from the University of California, consider a particular aspect of the collection process, namely, the collision efficiency of two spheres falling in a viscous medium (Collision Efficiencies of Two Spheres Falling in a Viscous Medium for Reynolds Numbers up to 19.2. Pp. ix +140 . Berkeley and Los Angeles: University of California Press; London: Cambridge University Press, 1964. 3 dollars). At a given instant and for a given size distribution of droplets in a cloud, there is a definite distribution of fall velocities of the droplets, and relative to the air the larger droplets (drops) fall more rapidly than the smaller droplets. For the collection of a droplet by a drop, the two have not only to collide but also to coalesce. The coalescence will depend on several factors such as impact speed and angle, the surface tension and electrical polarity of the droplet and drop, and on the physical properties of their environment. The exact determination of the collection efficiency in a cloud is therefore a complicated problem, but the basic process is that of two spheres falling in a viscous medium, and the computation of the theoretical collision efficiency has been treated by several investigators for particular cases.
For example. Hocking considered both spheres to be small $\left(R_{\Theta}<0.44\right.$ corresponding to a radius of the larger sphere of about $<30 \mu$ for water droplets in air) so that the non-linear terms in the equations of motion of the fluid could be neglected. His results, which are shown graphically, are taken by Shafrir and Neiburger as a reference for the range of small Reynolds numbers $(R \theta<0.44)$ where the Stokes's approximation is valid. For larger Reynolds numbers, Hocking's method is not applicable. and Shafrir and Neiburger base their computation on the assumption that an accurate numerical solution of the equations of fluid motion around, one sphere, including all the non-linear terms, can furnish the approximate drag force on the other sphere in a relatively simple form. The disturbance influencing the drag force on each sphere is taken to depend on the flow round the other sphere alone, and the equations of motion of the spheres are then integrated numerically to obtain the grazing trajectories.

In the first two chapters, a description is given of the equations of motion and of their integration, and of the computation of the collision efficiencies. The flow fields, including the non-linear effects, are evaluated using a modification of the method employed by Jensen, and the numerical work, which was porformed with the aid of an IBM 7090 digital computer, is fully described in the appendix to the article. Tables of numerical values for the vorticity fields and stream function cover some 75 pages, and the values are given to three decimal places for the range of $R \Theta=0.9677$ to $19 \cdot 1936$, that is for water-drops of radii $40 \mu-136 \mu$, falling in air at 900 mbar pressure and $0^{\circ} \mathrm{C}$ temperature.

\section{OCCUPATIONAL VISION SCREENING}

\begin{abstract}
A RECENT survey by Paul E. Ungar in an electronic equipment manufacturing company (Occupational Health, 16, No. 6, 1964) showed that 30 per cent of the employees had a significant, uncorrected visual defectthis in a factory where good oyesight is an asset to speedy and accurate production. About half the employees examined already wore spectacles, but they were often inadequate. Of the 56 employees who failed the screening tests, 35 did so wearing their spectacles. Similar surveys carried out in other plants revealed much the same state
\end{abstract}

of affairs; in soms instances the incidence of visual defects was as high as 40 per cent.

Even to-day, the most widely used method of checking on the eyesight of employees is the Snellen letter chart. This can only measure monocular distance visual acuity. Sometimes - but by no means always-a near vision test also is given, using one of the several test types available for this purpose, such as Jaeger's test types. Where good colour vision is required, it is most frequently tested with the Ishihara colour test plates. Safoty, as 
well as job performance, has to be borne in mind now that many hazards are indicated by colour codes. These test progedures have a number of shortcomings. Tests assess what each eye is capable of seoing with its fellow occluded. Often this is something different from what one eye will see when the other eye is open, as is the case under ordinary working conditions. Moreover, these tests are based on the subject's ability to recognize and interpret letters or words, whereas, occupationally, a more competent test is one incorporating the need for correct recognition of form or pattern such as fine criss-cross lines or dots. This is equally true for persons engaged in clerical work, for which acuity tests using such patterns are also generally more valid. The Ishihara plates are indeed most useful so long as they are used under the type of illumination for which the particular book of plates has been designed.

The testing of distance and near visual acuity and colour vision, however, is not in itself sufficient for a complete assessment of a person's visual fitness and suitability for his or her job. Many who have perfectly clear distance and near vision may eomplain of headaches, lack of concentration or eye-strain. They may lack accuracy and speod in the performance of their work. They may have difficulty in using instruments such as binocular microseopes. Any and all of these problems may be due to defects of 'binocular vision'. If the two eyes do not work properly together as a well-matched pair, if there is disturbance in the muscle balance or eye co-ordination, fatigue with all its consequences may result, faulty visual judgments may be made and the sufferer may become more accident-prone. Side field of vision can also be of considerable importance, as in the case of drivers, truck and crane operators. Normally termed 'vision screening', a comprehensive өy $\theta$ check is a practical proposition in every factory, office or store, with modern equipment specifically designed for the purpose.

A 'vision screener' is a compact, entirely self-contained optical instrument of high precision. It is basically a stereoscopic device and all kinds of binocular tests can be performed with it. An ingenious application of the principles of stereoscope design makes it unnecessary ever to occlude one eye-even when testing monocular visual acuity. These instruments have been designed so that they can easily be operated. It should be emphasized, however, that no machine can be a substitute for an eye examination by a qualified ophthalmic practitioner.

\title{
VAN DER WAALS INTERACTION AND THE STABILITY OF HELICAL POLYPEPTIDE CHAINS
}

\author{
By P. DE SANTIS, E. GIGLIO, A. M. LIQUORI and A. RIPAMONTI \\ Istituto Chimico, Università, di Napoli, Napoli (Italy) and Centro Nazionale di Chimica delle Macromolecole \\ (CNR) Sez. III
}

$\mathrm{W}^{\mathrm{i}}$ E have previously reported ${ }^{1-4}$ analyses of the geometry and relative stability of helical macromolecules consisting of repetitions of equivalent skeleton bonds $(A-A-A$. . . chains) and of pairs of skeleton bonds $(A-B-A-B \ldots$ chains). We calculated the conformational potential energy of helices as a function of the angles of rotation about the skeleton bonds of a monomer unit. To do this, we postulated structural equivalence of monomers along the fibre axis and used semi-empirical potential functions to describe the pairwise van der Waals interactions between 'non-bonded' atoms in the chain. Conventional bond-lengths and bond-angles were used.

The angles of rotation of the most stable helices were obtained by locating the deepest minima of the conformational potential energy in one- and two-dimensional plots for $A-A$ and $A-B$ chains respectively. The corresponding helical parameters, namely, the number of monomers per turn $K$ and the monomer repeat on the helical axis $d$, were then derived from the angles of rotation following a matrix method ${ }^{2,4,5}$.

The parameters, so calculated, agreed with those derived $^{2-4}$ from the interpretation of fibre diffraction photographs for all the polymers considered, showing that this method can predict the most stable helices of linear macromolecules with a simple skeleton from van der Waals interactions between 'non-bonded' atoms.

This approach has now been extended to polypeptide chains which should be formally considered as $A-B-C$ chains but, taking into account the rigid nature of the amide group, may be treated either as $A-B$ chains (polyglycine and poly-x- $\alpha$-alanine) or $A-A$ chains (cis and trans poly-L-proline).

Van der Waals conformational potential energy of helical polypeptides. The conformational potential energy of helical polypeptide chains was calculated as a function of the angles of rotation about the non-rigid skeleton bonds of an amino-acid residue, using the set of potential functions ${ }^{4}$ shown in Table 1 to describe the van dex Waals interactions between 'non-bonded' atoms. Bondlengths and bond-angles were fixed according to the values of Corey and Pauling.

The two-dimensional plot of the conformational potential energy of polyglycine contains three minima which will be referred to as I, II, III in the order of increasing energy values (Fig. 1). The angles of rotation about the skeleton bonds of the stable structures corre sponding to these minima are listed in Table 2 together with the helical parameters. Structure II is an $\alpha$-helix. Structure $I$ is similar to the three-fold helix proposed by Crick and Rich $^{2}$ from the X-ray diffraction photograph

Table 1. Potentral FunCtions of PAIRwrse INTERACTION BETWEEN

Interaction Interaction energy ${ }^{3}$ in kcal/atom pair (when $r$ is in $A$ )

$\mathrm{H} \ldots \mathrm{H}$

o....

$3.726 \times 10^{3} \times \exp (-3.070 r)-89.52 \times r^{-6}$

N.... N

$\mathrm{CH}_{2} \ldots . \mathrm{CH}_{3}$

$1.057 \times 10^{5} \exp (-4.608 r)-125.1 \times r^{-6}$

$(1 \cdot 7<r<2 \cdot 30 \AA)$

$2 \cdot 390 \times 10^{4} \times r^{-7 \cdot 37}$

$(r>2 \cdot 30 \AA)$

$(r<3.20 \AA)$

$2.739 \times 10^{5} \times \exp (-3.329 r)-2942 \times r^{-8} \quad(r>3.20 \AA)$

$\mathrm{C} \ldots \mathrm{C}$

C.... H*

$012 \times 10^{5} \times r^{-12}-327 \cdot 2 r^{-6}$

$2.879 \times 10^{5} \times \exp (-1.664 r)-1,235 r^{-6}$

$\mathrm{H} . \ldots \mathrm{CH}_{3}{ }^{*} \quad 3.190 \times 10^{4} \times \exp (-3.199 r)-2,089 \times r^{-6}$

O . . . H H $\quad 1.982 \times 10^{4} \times \exp (-3.839 r)-497.6 \times r^{-8}$

$\mathrm{N} \ldots \mathrm{H}$

O . . . $\mathrm{CH}_{8}{ }^{*} \quad 1.702 \times 10^{5} \times \exp (-3.969 r)-796-2 r^{-6}$

$\mathrm{N} \ldots \mathrm{CH}_{3}$

$\mathrm{O} . . . \mathrm{C}^{*}$

$\mathrm{N} \ldots \mathrm{C}$

$1 \cdot 785 \times 10^{5} \times \exp (-2 \cdot 304 r)-202 \cdot 2 \quad r^{-8}$

* The mixed interactions were minimized using the following values of the van der Waals radii: $R_{\mathrm{H}}=1 \cdot 20 \AA, R_{\mathrm{C}}=1.70 \AA ; R_{\mathrm{O}}=2.00 \AA ; R_{0}=1.35 \AA$; $R_{\mathrm{H}}=1.35 \AA$. 